PROCEEDINGS OF THE

AMERICAN MATHEMATICAL SOCIETY

Volume 130, Number 7, Pages 2037-2043

S 0002-9939(02)06469-9

Article electronically published on February 12, 2002

\title{
ON THE REDUCIBILITY OF CHARACTERISTIC VARIETIES
}

\author{
TOM BRADEN
}

(Communicated by Michael Stillman)

\begin{abstract}
We show that some monodromies in the Morse local systems of a conically stratified perverse sheaf imply that other Morse local systems for smaller strata do not vanish. This result is then used to explain the examples of reducible characteristic varieties of Schubert varieties given by Kashiwara and Saito in type $A$ and by Boe and Fu for the Lagrangian Grassmannian.
\end{abstract}

Let $X=G / P$ be a flag variety associated to a complex semisimple group $G$ and parabolic subgroup $P$. Let $Y \subset X$ be a Schubert variety, the closure of an orbit under the action of a Borel subgroup $B \subset G$ on $X$. The characteristic cycle $C C(\mathbf{I C} \cdot(Y))$ of the intersection cohomology sheaf on $Y$ is an object of considerable representation theoretic interest. It is an $n$-dimensional closed-support cycle (where $\left.n=\operatorname{dim}_{\mathbb{R}} X\right)$ supported on the conormal variety $\Lambda \subset T^{*} X$ to the Schubert stratification. Since $\Lambda$ is purely $n$-dimensional, with one component for each stratum, this amounts to giving an integer for each Schubert cell.

The support of the characteristic cycle is known as the characteristic variety. In 9], Kazhdan and Lusztig stated a conjecture equivalent to the statement that for $G=S L_{n}$, the characteristic varieties of intersection cohomology sheaves should be irreducible. This work arose from an attempt to understand the counterexample found by Kashiwara and Saito 7] to this conjecture.

We present a theorem (Theorem 2 see also Corollary 3) which explains many of the examples currently known (for general semisimple $G$, not just $G=S L_{n}$ ) in which $C C(\mathbf{I C}(Y))$ is reducible. It gives a geometric condition on a stratified variety $Y$ and a stratum $S$ that implies that the component $\left[\Lambda_{S}\right]$ lying over $S$ appears in $C C(\mathbf{I C} \cdot(Y))$; it requires that $Y$ have a conical singularity along $S$.

We use this result to verify Kashiwara and Saito's example, and to explain the examples of non-reducible characteristic varieties arising in Lagrangian Grassmannians found by Boe and $\mathrm{Fu}[1]$. Our result can also be used to explain all but one of the examples found by Tanisaki [12] of reducible characteristic varieties in full flag manifolds. Our result cannot show that components of $\Lambda$ do not occur in the characteristic variety, nor does it provide a way to calculate the actual multiplicities, as both Boe and $\mathrm{Fu}$ and Tanisaki were able to do.

We take $\mathbb{Q}$ as our ground field throughout this paper; all intersection cohomology sheaves are taken with constant $\mathbb{Q}$ coefficients.

Received by the editors February 27, 2000 and, in revised form, January 29, 2001.

2000 Mathematics Subject Classification. Primary 32S60; Secondary 32S30.

Key words and phrases. Perverse sheaves, vanishing cycles, Morse group, characteristic variety. 


\section{VANISHING CYCLES OF CONICAL SHEAVES}

This section presents an argument from [2]. Let $V$ be a complex vector space, and suppose an object $\mathbf{A}$ in the derived category $D^{b}(V)$ is constructible with respect to an algebraic, $\mathbb{C}^{*}$-conical stratification. Let $f: V \rightarrow \mathbb{C}$ be a linear function, and let $W=f^{-1}(0)$. For definitions of the sheaf-theoretic concepts that we use, including the vanishing cycles functor $\phi_{f}$ and the Fourier transform $F$, see 8 .

Theorem 1. The multiplicity of $\left[T_{\{0\}}^{*} V\right]$ in $C C(\mathbf{A})$ is the same as the multiplicity of $\left[T_{\{0\}}^{*} W\right]$ in $C C\left(\phi_{f} \mathbf{A}\right)$.

Proof. The Fourier transform $F: D^{b}(V) \rightarrow D^{b}\left(V^{*}\right)$ respects the characteristic cycle: we have $C C(F \mathbf{A})=C C(\mathbf{A})$, using the natural identification $T^{*} V \cong V \times V^{*} \cong$ $T^{*} V^{*}$ (this is given as Exercise 9.7 in [8]). Since the multiplicity of the zero section $T_{V^{*}}^{*} V^{*}$ in $C C(F \mathbf{A})$ is the Euler characteristic of the stalk cohomology of $\mathbf{A}$ at a point in the open stratum, it will be enough to show that for generic inclusions $i_{1}$, $i_{2}$ of a point $\{p\}$ into $V^{*}$ and $W^{*}$, respectively, the restrictions $i_{1}^{*} F \mathbf{A}$ and $i_{2}^{*} F \phi_{f} \mathbf{A}$ are isomorphic.

We can consider $f$ as an element of $W^{\perp} \subset V^{*}$. Let $s: W \rightarrow W \times W^{\perp}$ be given by $s(w)=(w, f)$. Then $\phi_{f}$ is naturally isomorphic to the composition of the top row in the following diagram:

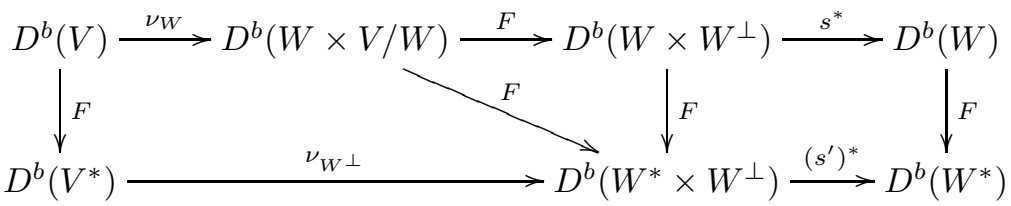

Here $\nu_{W}, \nu_{W} \perp$ are the specialization functors (using the natural identifications $T_{W} V \cong W \times V / W$ and $\left.T_{W \perp} \cong W^{\perp} \times W^{*}\right)$, the functors marked $F$ are the appropriate Fourier transforms, and $s^{\prime}(\omega)=(\omega, f)$. The left quadrilateral and the triangle naturally commute by [4, Propositions 2.3 and 2.4, and the right-hand square commutes by [8], Proposition 3.7.13.

Thus $F \phi_{f} \mathbf{A} \cong\left(s^{\prime}\right)^{*} \nu_{W} F \mathbf{A}$. It is now easy to see that $\nu_{W \perp}$ and $\left(s^{\prime}\right)^{*}$ both leave the dimensions of the stalk cohomology at a generic point unchanged (for $\left(s^{\prime}\right)^{*}$, use the fact that $\nu_{W} \perp F \mathbf{A}$ is conical).

Similar techniques have been useful in the calculation of certain categories of perverse sheaves [3], 4].

\section{THE MAIN RESUlT}

Suppose a smooth complex variety $V$ carries an algebraic Whitney stratification $\mathcal{S}$. Denote the conormal variety by $\Lambda \subset T^{*} V$, and let $\Lambda_{S}=\overline{T_{S}^{*} V}$ be the component of $\Lambda$ lying over $S \in \mathcal{S}$. Let $\widetilde{\Lambda}$ be the smooth part of $\Lambda$, and for each stratum $S$ put $\widetilde{\Lambda}_{S}=\widetilde{\Lambda} \cap \Lambda_{S}$.

Definition. If two strata $S$ and $T$ satisfy $\operatorname{dim}_{\mathbb{C}}\left(\Lambda_{S} \cap \Lambda_{T}\right)=\operatorname{dim}_{\mathbb{C}} \Lambda-1$, we say that $S$ and $T$ meet microlocally in codimension one.

Given an $\mathcal{S}$-constructible perverse sheaf $\mathbf{P}$ on $V$, we let $\mathcal{M}_{S}(\mathbf{P})$ be the Morse or vanishing cycles local system of $\mathbf{P}$ at the stratum $S$ (for a definition, see [10]). It 
is a local system on $\widetilde{\Lambda}_{S}$, whose dimension is, up to a sign, the multiplicity of $\left[\Lambda_{S}\right]$ in $C C(\mathbf{P})$.

Now suppose that $V$ is a complex vector space and the stratification $\mathcal{S}$ is $\mathbb{C}^{*}$ conical. Let $S \neq\{0\}$ be a stratum, and take a point $x \in S$. Define a loop $\gamma$ in $\widetilde{\Lambda}_{S}$ by choosing a point $(x, \xi) \in \widetilde{\Lambda}_{S}$ and letting $\gamma(\theta)=\left(e^{i \theta}, \xi\right), \theta \in[0,2 \pi]$. Note that any two such loops are homotopic.

Theorem 2. Suppose that $\mathbf{P}$ is an $\mathcal{S}$-constructible perverse sheaf on $V$. If $S$ meets $\{0\}$ microlocally in codimension one, and the monodromy of $\mathcal{M}_{S}(\mathbf{P})$ around $\gamma$ is not multiplication by $(-1)^{d-1}$, then $\mathcal{M}_{0}(\mathbf{P}) \neq 0$.

In the case $\mathbf{P}=\mathbf{I C}(\bar{S})$, we get:

Corollary 3. Suppose $X \subset V$ is an even-dimensional conical variety, $S$ is the smooth locus of $X$, and $\{0\}$ and $S$ meet microlocally in codimension one. Then $\mathcal{M}_{0}(\mathbf{I C} \cdot(X)) \neq 0$.

Proof. Just note that $\mathcal{M}_{S}(\mathbf{I C}(\bar{S})) \cong \mathbb{Q}_{\tilde{\Lambda}_{S}}$.

Proof of Theorem 2. Since we are free to choose the point $(x, \xi) \in \widetilde{\Lambda}_{S}$, we choose it as follows. The dual cone $S^{\vee} \subset V^{*} \cong T_{0}^{*} V$ to $S$ is defined to be $\Lambda_{S} \cap T_{0}^{*} V ; S$ and $S^{\vee}$ are cones over dual projective varieties in $\mathbb{P} V$ and $\mathbb{P} V^{*}$. By assumption $S^{\vee}$ is a divisor and so it is not contained in any $T^{\vee}$ for any stratum $T \neq S,\{0\}$. Let $\xi$ be a smooth point of $S^{\vee} \backslash S^{\vee} \cap \bigcup_{R \neq S,\{0\}} R^{\vee}$. Then $L=\{v \in V \mid(v, \xi) \in \Lambda\}$ is a line through the origin contained in $S \cup\{0\}$; let $x$ be any nonzero point in $L$.

In microlocal language, the point $(0, \xi)$ represents a codimension one point of $\Lambda$ : it is a smooth point of both $\Lambda_{0}$ and $\Lambda_{S}$, they intersect transversely there, and it lies in no other component of $\Lambda$.

Let $f: V \rightarrow \mathbb{C}$ be the linear function with $d f_{x}=\xi$. Our choice of $\xi$ implies that $\left.f\right|_{S}$ has singularities only on $L$ and that if we choose a normal slice $N$ to $L$ at $x \in L$, the singularity of $\left.f\right|_{N \cap S}$ is Morse at $x$.

Let $L^{\circ}=L \backslash\{0\}$ and embed $L^{\circ}$ into $\widetilde{\Lambda}$ by the map $\alpha(y)=\left(y, d f_{y}\right)=(y, \xi)$. Put $\mathbf{P}_{f}=\phi_{f}(\mathbf{P})$; it is a conical perverse sheaf supported on $L$. The local system on $L^{\circ}$ can be computed using stratified Morse theory:

$$
\left.\mathbf{P}_{f}\right|_{L^{\circ}}=\alpha^{*} \mathcal{M}_{S}(\mathbf{P}) \otimes \mathcal{L}
$$

where $\mathcal{L}=\phi_{\left(\left.f\right|_{S}\right)}\left(\mathbb{C}_{S}\right)$. But the local system $\mathcal{L}$ is easy to compute by PicardLefchetz theory; its monodromy is multiplication by $(-1)^{d-1}$, where $d=\operatorname{dim}_{\mathbb{C}}(S)$. Thus the monodromy of $\left.\mathbf{P}_{f}\right|_{L^{\circ}}$ is nontrivial.

An elementary application of the theory of perverse sheaves on a complex line (see 13 and the example following the proof of Theorem 3.3 in [10]) implies that a perverse sheaf with nontrivial monodromy around the origin must have a nonzero vanishing cycle at 0 ; thus $\mathcal{M}_{0}\left(\mathbf{P}_{f}\right) \neq 0$. Then by Theorem $1, \mathcal{M}_{0}(\mathbf{P})=\mathcal{M}_{0}\left(\mathbf{P}_{f}\right)$, so we are done.

Remark. A strengthening of Theorem 1 using Morse local systems instead of characteristic cycles gives the slightly stronger conclusion that $\mathcal{M}_{0}(\mathbf{P})$ is a nontrivial local system.

We have stated Theorem 2 assuming that the ambient variety is a vector space and the smaller stratum is a point, but it can also be applied to a pair of strata $(S, T)$ in a general stratified complex manifold $V$ with $T \subset \bar{S}$, so long as the stratification 
is conical along $T$; simply intersect with a normal slice $N$ to $S$ at a point $s \in S$, or, equivalently, apply the specialization functor $\nu_{S}: D^{b}(V) \rightarrow D^{b}\left(T_{S} V\right)$ and restrict to the fiber over $s$.

If $V \cong \mathbb{C}^{n}$ but the stratification of $V$ is not conical, one can still look at the specialization $\mathbf{P}^{\prime}=\nu_{0}(\mathbf{P})$, which is conical. Since $\mathcal{M}_{0}\left(\mathbf{P}^{\prime}\right) \cong \mathcal{M}_{0}(\mathbf{P})$ (see [11), Theorem 2 may apply to $\mathbf{P}^{\prime}$ to give information about $\mathbf{P}$. We cannot use this to deduce that Theorem 2 holds without the conical assumption, however. There are stratifications for which $S$ meets 0 microlocally in codimension one, but the smooth part of the specialization $\nu_{0}(\bar{S})$ doesn't.

For instance, if $\bar{S}$ is the variety $\left\{(x, y, z) \in \mathbb{C}^{3} \mid x y=z^{3}\right\}$, the specialization $\mathbf{P}^{\prime}=\nu_{S}(\mathbf{I C} \cdot(\bar{S}))$ is supported on $\{x y=0\}$. A little calculation shows that the composition series of the perverse sheaf $\mathbf{P}^{\prime}$ consists of simple intersection cohomology sheaves with constant coefficients supported on $\{x=0\},\{y=0\},\{x=y=0\}$, and $\{(0,0,0)\}$. If Theorem 2 could be applied to $\mathbf{P}^{\prime}$, it would have to apply for one of these simple components, since the Morse local systems $\mathcal{M}_{S}$ functors are exact on the category of perverse sheaves. But this is clearly not the case, since all but the last have $\mathcal{M}_{0}=0$.

This singularity appears in the flag variety for $G=G_{2}$, associated to the pair of Weyl group elements $(t s t, t s t s t)$, where $s, t$ are the reflections for the long and short simple roots, respectively.

\section{Kashiwara And Saito's EXAmple}

In 77. Kashiwara and Saito gave an example of a pair of Schubert varieties $Z \subset$ $Y$ in the variety of complete flags in $\mathbb{C}^{8}$ so that $\Lambda_{Z}$ appears in the support of $C C(\mathbf{I C}(Y))$. We recall their description of a normal slice to $Y$ at a generic point of $Z$. Let $V \cong \mathbb{C}^{16}$ be the space $\operatorname{Mat}(2 \times 2, \mathbb{C})^{4}$ of 4 -tuples of $2 \times 2$ complex matrices. Let $X$ be the variety

$$
\left\{\left(A_{0}, A_{1}, A_{2}, A_{3}\right) \in V \mid \operatorname{det}\left(A_{i}\right)=0 \text { and } A_{i} A_{i+1}=0 \text { for all } i\right\},
$$

where the index $i$ is taken modulo 4 . It is an 8-dimensional conical subvariety of $V$.

We show that $X$ satisfies the hypotheses of Corollary 3 Let $G=\left(G L_{2}(\mathbb{C})\right)^{4} \times \mathbb{C}^{*}$ act on $V$ by

$$
\left(g_{0}, g_{1}, g_{2}, g_{3}, t\right) \cdot\left(A_{0}, A_{1}, A_{2}, A_{3}\right)=\left(t g_{0} A_{0} g_{1}^{-1}, g_{1} A_{1} g_{2}^{-1}, g_{2} A_{2} g_{3}^{-1}, g_{3} A_{3} g_{0}^{-1}\right) .
$$

Then $X$ is $G$-invariant, and in fact is the closure of a $G$-orbit, $X=\overline{G \cdot(A, A, A, A)}$, where $A$ is any nonzero nilpotent matrix. The open orbit is the subset of points $\left(A_{0}, A_{1}, A_{2}, A_{3}\right)$ in $X$ for which all the $A_{i}$ are nonzero.

Proposition 4. The smooth part of $X$ meets $\{0\}$ microlocally in codimension one.

Proof. This is equivalent to showing that the dual cone $X^{\vee} \subset V^{*}$ is a divisor. Using the inner product on $V$ given by matrix coordinates, $V^{*}$ is naturally identified again with $\operatorname{Mat}(2 \times 2, \mathbb{C})^{4}$, with the action of $G$ given by

$$
\left(g_{0}, g_{1}, g_{2}, g_{3}, t\right) \cdot\left(A_{0}, A_{1}, A_{2}, A_{3}\right)=\left(t^{-1} g_{1} A_{0} g_{0}^{-1}, g_{2} A_{1} g_{1}^{-1}, g_{3} A_{2} g_{2}^{-1}, g_{0} A_{3} g_{3}^{-1}\right) .
$$

Certainly $X^{\vee}$ is $G$-stable, and it is easily checked that $x=\left(I+A^{t}, I, I, I\right)$ lies in $X^{\vee}$, where again $A$ is any nonzero nilpotent matrix. Its stabilizer is $G_{x}=\{(g, g, g, g, 1)\}$ $\left.g=a I+b A^{t}, a \neq 0\right\}$, a two-dimensional group, so $\operatorname{dim}_{\mathbb{C}} X^{\vee}=17-2=15$. 
Thus Corollary 3 applies to this example, showing that $\left[\Lambda_{\{0\}}\right]$ appears with nonzero multiplicity in $C C\left(\mathbf{I C}^{*}(X)\right)$.

Also note that $X$ is a toric variety: although the maximal torus $T \subset S L_{8}(\mathbb{C})$ which acts on the flag variety is only 7 -dimensional, there is a larger torus in $G$ which acts on $X$ with finitely many orbits. A laborious calculation with an algorithm from [2] or the equivalent formula in [5] (Theorem 2.12 in Chapter 10) shows that $\operatorname{dim} \mathcal{M}_{0}(\mathbf{I C}(X))=1$.

\section{The Lagrangian Grassmannian}

In [1, Boe and Fu computed the characteristic cycles $C C(\mathbf{I C}(Y))$ for Schubert varieties $Y$ in Hermitian symmetric spaces. In the compact cases, reducible characteristic varieties appeared only for the Lagrangian Grassmannian $X$ of Lagrangian subspaces of a complex symplectic space $\mathbb{C}^{2 n}$. For concreteness, suppose that the symplectic form is given by $\omega=\sum_{i=1}^{n} \mathbf{e}_{i}^{*} \wedge \mathbf{e}_{2 n+1-i}^{*}$, where the $\mathbf{e}_{i}^{*}$ form the dual basis to the standard basis of $\mathbb{C}^{2 n}$.

The Schubert decomposition of $X$ is given as follows. Let $B \subset \operatorname{Sp}\left(\mathbb{C}^{2 n}, \omega\right)$ be the Borel group of transformations preserving the standard flag. Given a word $w \in\{\alpha, \beta\}^{n}$ of length $n$ in the letters $\alpha$ and $\beta$, we define a cell $S_{w}$ as follows. Let $\bar{w} \in\{\alpha, \beta\}^{2 n}$ be the word for which $\bar{w}(2 n+1-i) \neq \bar{w}(i)=w(i)$ for $1 \leq i \leq n$. Then $E_{w}=\operatorname{span}\left\{\mathbf{e}_{i} \mid \bar{w}(i)=\alpha\right\}$ is a point in $X$, and we put $S_{w}=B \cdot E_{w}$ and $X_{w}=\overline{S_{w}}$. Also let $N_{w}=B^{-} E_{w}$, where $B^{-}$is the opposite Borel to $B$; it is a normal slice to $S_{w}$ through $E_{w}$.

Let $\mathbf{P}_{w}=\mathbf{I C}\left(X_{w}\right)$. Given $v, w \in\{\alpha, \beta\}^{n}$, let $m_{v}^{w}$ be the multiplicity with which $\left[\Lambda_{v}\right]$ appears in $C C\left(\mathbf{P}_{w}\right)$. Boe and Fu's calculation of these numbers can be summarized as follows.

Theorem 5 (Boe and Fu [1], Theorem 7.1D). $m_{v}^{w}=0$ or 1 for any $v, w \in\{\alpha, \beta\}^{n}$. It is 1 if and only if there is a chain

$$
X_{w}=X_{1} \supset X_{2} \supset \cdots \supset X_{n}=X_{v}
$$

of Schubert varieties $X_{i}=X_{w_{i}}$ so that for all $1 \leq i<n$ the codimension $\operatorname{dim}_{\mathbb{C}} X_{i}-$ $\operatorname{dim}_{\mathbb{C}} X_{i+1}$ is even and $S_{w_{i}}$ meets $S_{w_{i+1}}$ microlocally in codimension one.

Suppose that there exists a chain $\left\{w_{i}\right\}$ satisfying the conditions of this theorem. We will use Theorem 2 to give a shorter proof that $m_{v}^{w} \neq 0$. Let $E_{i}=E_{w_{i}}$, $S_{i}=S_{w_{i}}, \widetilde{\Lambda}_{i}=\widetilde{\Lambda}_{S_{i}}$. Assume inductively that $m_{w_{i}}^{w}>0$ (this is trivially true for $i=1)$, so $\mathcal{M}_{i}=\mathcal{M}_{S_{i}}\left(\mathbf{P}_{w}\right)$ is a nonzero local system on $\widetilde{\Lambda}_{i}$. We will show that this implies $\mathcal{M}_{i+1}$ is nonzero as well.

Put $E=E_{i+1}, N=N_{w_{i+1}}$, and $S=N \cap S_{i}$ and let

$$
\mathbf{Q}=\left.\mathbf{P}_{w}\right|_{N}\left[-\operatorname{dim}_{\mathbb{C}} S_{i}\right] \cong \mathbf{I C}\left(X_{w} \cap N\right) .
$$

The degree shift makes $\mathbf{Q}$ a perverse sheaf.

We give $N$ the stratification induced from $\left\{S_{w}\right\}$. Let $\gamma$ be a loop in $\widetilde{\Lambda}_{S}$ as in Theorem 2 We will show that the local system $\mathcal{M}_{S}(\mathbf{Q})$ has trivial monodromy around $\gamma$, or equivalently, that $\mathcal{M}_{i}$ has trivial monodromy around $\rho \circ \gamma$, where $\rho: \Lambda_{N} \rightarrow \Lambda$ is the inverse to the homeomorphism given by restricting the natural projection $\left.T^{*} X\right|_{N} \rightarrow T^{*} N$ to $\left.\Lambda \cap T^{*} X\right|_{N}$. We can then apply Theorem 2 to show that $\mathcal{M}_{\{0\}}(\mathbf{Q})=\mathcal{M}_{S_{i+1}}(\mathbf{P}) \neq 0$. 
There is an action of the torus $T=\left(\mathbb{C}^{*}\right)^{n+1}$ on $X$ which preserves the Schubert stratification, defined by acting on $\mathbb{C}^{2 n}$ via

$$
\left(z_{0}, z_{1}, \ldots, z_{n}\right) \cdot\left(x_{1}, \ldots, x_{2 n}\right)=\left(z_{1}^{-1} x_{1}, \ldots, z_{n}^{-1} x_{n}, z_{0} z_{n} x_{n+1}, \ldots, z_{0} z_{1} x_{2 n}\right) .
$$

The $E_{w}$ are all fixed points of this action, the normal slice $N$ is preserved, and the induced action on $N$ is linear. This action makes all Schubert variety singularities conical, as the following lemma shows.

Lemma 6. For any word $w \in\{\alpha, \beta\}^{n}$, there is a homomorphism $\chi_{w}: \mathbb{C}^{*} \rightarrow T$ for which the induced action of $\mathbb{C}^{*}$ on $N_{w}$ is the conical action.

Proof. Let $\chi_{w}(z)=\left(z, z^{a_{1}}, \ldots, z^{a_{n}}\right)$, where $a_{i}=-1$ if $w(i)=\beta$, and $a_{i}=0$ if $w(i)=\alpha$. Checking that this gives the required action is an exercise in local Grassmannian coordinates, similar to arguments in [1] and [3].

Proposition 7. The loop $\rho \circ \gamma$ is homotopic (in $\pi_{1}\left(\widetilde{\Lambda}_{i}\right)$ ) to a loop generated by the action of a loop in $\left(\mathbb{C}^{*}\right)^{n+1}$.

Proof. Acting on $\gamma$ by the loop $\theta \mapsto \chi_{w_{i+1}}\left(e^{-i \theta}\right)$, we obtain the loop $\chi_{w_{i+1}}^{-1} \cdot \gamma(\theta)=$ $\left(x, e^{i \theta} \xi\right)$, i.e. the point in $N$ stays fixed and the covector goes around by a conical action. We can then slide the loop $\rho \circ\left(\chi_{w_{i}}^{-1} \cdot \gamma\right)$ in $\widetilde{\Lambda}_{i}$ to get a loop of the form $\left(E_{i}, e^{i \theta} \xi^{\prime}\right)$ for some $\xi^{\prime} \in T_{E_{i}} X$. Acting now by $\chi_{w_{i}}\left(e^{i \theta}\right)$ now produces a trivial loop.

The fact that the monodromy of $\mathcal{M}_{i}$ around $\rho \circ \gamma$ is trivial now follows from the following proposition.

Proposition 8. Let $X$ be a complex variety with an action of a connected algebraic group $G$ and a $G$-invariant stratification. If $\mathbf{P}=\mathbf{I C} \cdot(\bar{S})$ for some stratum $S$, then the Morse local systems $\mathcal{M}_{T}(\mathbf{P})$ are constant along loops generated by acting by loops in $G$.

Proof. Since $\mathbf{P}$ is a $G$-equivariant perverse sheaf, the local systems $\mathcal{M}_{T}(\mathbf{P})$ must also be $G$-equivariant.

\section{REFERENCES}

[1] B. Boe and J. Fu, Characteristic cycles associated to Schubert varieties in classical Hermitian symmetric spaces, Canad. J. Math. 49 (1997), 417-467. MR 98j:14068

[2] T. Braden, Characteristic cycles of toric varieties; perverse sheaves on rank stratifications, MIT Ph.D. thesis, 1995.

[3] — Perverse sheaves on Grassmannians, preprint math.AG/9907152, to appear in Canadian J. Math.

[4] T. Braden and M. Grinberg, Perverse sheaves on rank stratifications, Duke Math. J. 96 (1999), 317-362. MR 2000f:14031

[5] I. Gel'fand, M. Kapranov, and A. Zelevinsky, Discriminants, resultants, and multidimensional determinants, Birkhäuser, 1994. MR 95e:14045

[6] M. Goresky and R. MacPherson, Stratified Morse Theory, Springer 1988. MR 90d:57039

[7] M. Kashiwara and Y. Saito, Geometric construction of crystal bases, Duke Math. J. 89 (1997), 9-36. MR 99e:17025

[8] M. Kashiwara and P. Schapira, Sheaves on Manifolds, Springer 1990. MR 92a:58132

[9] D. Kazhdan and G. Lusztig, A topological approach to Springer's representations, Adv. Math. 38 (1980), 222-228. MR 82f:20076

[10] R. D. MacPherson and K. Vilonen, Elementary construction of perverse sheaves, Inv. Math. 84 (1986), 403-435. MR 87m:32028 
[11] C. Sabbah, Quelques remarques sur la géométrie des espaces conormaux, Astérisque 130, 1985, 161-192. MR 87f:32031

[12] T. Tanisaki, Characteristic varieties of highest weight modules and primitive quotients, in "Representations of Lie groups, Kyoto, Hiroshima 1986", Adv. Stud. Pure Math. 14, Acad. Press 1988, pp. 1-30. MR 91b:17014

[13] J-L. Verdier, Prolongement des Faisceaux Pervers Monodromiques, Astérisque 130 (1985) 218-236. MR 87d:32019

Department of Mathematics and Statistics, University of Massachusetts-Amherst, Amherst, Massachusetts 01003

E-mail address: braden@math.umass.edu 